\title{
Women's health and kidney disease
}

The theme of this year's World Kidney Day — kidneys and women's health - highlights an important but often neglected area of nephrology and, more broadly, of global health. Chronic kidney disease (CKD) is underrecognized despite its negative impact on morbidity and mortality and huge societal costs. Even less attention is given to sex differences in nephrology research, despite evidence of sex-specific differences in kidney function and in the epidemiology, diagnosis, underlying mechanisms, natural history and outcomes of CKD, as well as the need to consider the reproductive health of affected women and the renal and wider health of their offspring. To raise awareness of these issues, Nature Reviews Nephrology presents a focus on women's health and kidney disease, published to coincide with World Kidney Day and International Women's Day.

Epidemiological studies suggest that predialysis CKD is more prevalent in women than in men, and women are more likely than men to be kidney donors, but more men than women receive dialysis or undergo kidney transplantation. The factors that contribute to these disparities are complex and likely involve biological differences that affect the rate of CKD progression and transplantation criteria (such as the presence of preformed antibodies), as well as sociocultural influences that affect access to care. In the first Review in this issue, Juan Jesus Carrero and colleagues collate data from across the globe to explore sex (that is, biological) and gender (that is, sociocultural) disparities in the epidemiology of CKD. These insights are important, not only to highlight differences in the prevalence and outcomes of kidney disease in men and women, but also to identify strategies to prevent and target CKD. Greater understanding of the biological mechanisms that underlie sex-specific differences in the prevalence and progression of CKD will potentially aid the identification of new treatment targets, whereas awareness of sociocultural influences might open avenues to be addressed by clinicians, policy makers and other communities.

Reproductive health and pregnancy are important issues for women with CKD and are explored in a second Review by Kate Wiles and colleagues. CKD is associated with reduced fertility and an increased risk of adverse pregnancy outcomes such as pre-eclampsia, preterm delivery and fetal growth restriction. Moreover, low birth weight is a risk factor for hypertension and kidney disease in later life ${ }^{1}$, perpetuating the cycle. In their Review, Wiles and colleagues discuss fertility issues and contraceptive options for patients with CKD. They describe the renal physiological changes that occur during pregnancy, the contribution of CKD and common comorbidities to pregnancy complications and approaches to the diagnosis and therapeutic management of CKD during pregnancy - an area of much needed research given that few studies have assessed drug safety in pregnant women.

Kidney function is closely linked to blood pressure control and cardiovascular health, and an accumulating body of literature demonstrates that sex-specific factors influence blood pressure, with consequential effects on kidney and cardiovascular function. The third Review in this focus issue by Katrina Mirabito Colafella and Kate Denton explores these sex-specific differences and describes the protective mechanisms of sex hormones on vasoregulatory pathways that are lost after menopause, and the sex hormone-independent effects of the sex chromosome complement on the transcription of genes that are involved in haemodynamic and immunomodulatory pathways. The findings described in this Review provide important insights into sex differences at the molecular, cellular and tissue levels that regulate blood pressure control. Although many questions remain unanswered, these findings emphasize the need to consider sex differences in preclinical and clinical studies to ensure that therapeutic targets are relevant across populations.

Finally, in a specially commissioned Viewpoint article, we asked four leading researchers from different geographical regions to discuss key considerations related to women's kidney health. They outline the specific risk factors and consequences of kidney disease in women, as well as policy issues, such as barriers to care and how these might be overcome, and suggest possible costeffective policies and/or systems that should be implemented to improve the kidney health of women and of future generations. Their responses highlight the need for further research into the kidney health of women and the inclusion of greater numbers of women in CKD clinical trials as well as a general requirement for greater awareness and education relating to sex disparities in renal health and issues specific to renal health in pregnancy. Ultimately, these goals bring us full circle to the objectives of the 13th World Kidney Day campaign, which are to promote affordable and equitable access to health education, health care and prevention of kidney diseases for all women and girls worldwide. We hope this focus issue will increase awareness of the considerations and challenges specific to the renal health of women, as well as encourage further research into this important area.

Luyckx, V. A. \& Brenner, B. M. Birth weight, malnutrition and kidney-associated outcomes - a global concern. Nat. Rev. Nephrol. 11, 135-149 (2015). 